\title{
Efectos del almacenamiento y de la época de aplicación del nitrógeno sobre la cobertura foliar y el rendimiento en papa (Solanum tuberosum L.)
}

D. O. Caldiz; C. Della Croce; E. Vera; L. del Carmen Marchan*

\section{RESUMEN}

Se estudió la influencia del sistema de almacenamiento y de la época de aplicación del nitrógeno $(\mathrm{N})$ sobre la capacidad de cobertura foliar (CCF) y el rendimiento en los cultivares Spunta y Ballenera MAA, de maduración semitemprana y semitardía respectivamente. Los tubérculos fueron almacenados a $4^{\circ} \mathrm{C}$ en oscuridad $(F)$ y a temperatura ambiente en luz natural difusa (LND) durante 100 y 120 días en 1985-86 y 1986-87, respectivamente. El N se aplicó en forma de urea: testigo $\left(\mathrm{N}_{0}\right) ; 200 \mathrm{Kg}$./ha de $\mathrm{N}$ a la plantación $\left(\mathrm{N}_{1}\right)$ y fraccionado: $100 \mathrm{Kg}$./ha a la plantación y $100 \mathrm{Kg}$./ha al inicio de la tuberización $\left(\mathrm{N}_{2}\right)$. En 1985-86 la aplicación de $\mathrm{N}$ no influyó significativamente en el rendimiento. En 1986-87 la aplicación de nitrógeno fraccionado $\left(\mathrm{N}_{2}\right)$, incrementó la CCF hacia el final del ciclo de cultivo. Las modificaciones en el CCF estuvieron más relacionadas con las aplicaciones de nitrógeno que con el sistema de almacenamiento utilizado o el cultivar. Sólo en el cv. Spunta se registró un aumento en el rendimiento por la aplicación de nitrógeno fraccionado $\left(\mathrm{N}_{2}\right)$, cuando el material había sido conservado en LND.

Palabras Claves Adicionales: Capacidad de cobertura foliar, edad fisiológica, emergencia, nitrógeno.

Aceptado para publicación: Julio 4, 1989

\footnotetext{
* Trabajo realizado dentro del Programa "Ecofisiología de Papa", Ministerio de Asuntos Agrarios de la Provincia de Buenos Aires - Instituto de Fisiología Vegetal, Facultad de Agronomía, UNLP. C.C. 31, 1900 La Plata, Argentina.
} 


\section{ABSTRACT \\ Effects of Storage System and Time of Nitrogen Application on Foliage Cover Capacity and Yield in Potatoes}

It were studied the influences of different storage system and time of Nitrogen applications on the foliage cover capacity and yield of two cultivars, Spunta and Ballenera MAA, medium-early and medium-late types, respectively. Seed tubers were stored at $4^{\circ} \mathrm{C}(\mathrm{F})$ and at ambient temperature under natural diffuse light (LND) for 100 and 120 days in 1985-86 and 1986-87 respectively. Nitrogen was applied as urea; treatments compared were $\mathrm{N}_{0}$ : Control without nitrogen: $\mathrm{N}_{1}: 200$ $\mathrm{kg} / \mathrm{ha} \mathrm{N}$ at planting and $\mathrm{N}_{2}$ : fractioned, $100 \mathrm{~kg} / \mathrm{ha}$ at planting, 100 $\mathrm{kg} / \mathrm{ha}$ at tuber initiation. In 1985-86, experiments showed that $\mathrm{N}$ applications did not influence on tuber yield. In 1986-87, however, the fractioned application of $N_{1}$ increased foliage cover capacity by the end of crop cycle. Modifications in foliage cover capacity were more related to $\mathrm{N}$ applications than to storage systems or cultivars. Only cv. Spunta registered a yield increase by $\mathrm{N}_{2}$ when seed tubers were physiologically old.

Additional Index Words: Foliage cover capacity, physiological age, emergence, Nitrogen.

La producción de tubérculo-semilla requiere de la adopción de una tecnología particular, respecto a los aspectos sanitarios, las condiciones de cultivo y de almacenamiento. En los últimos años, en Argentina, se han logrado mejoras en la producción debido al aporte del mejoramiento genético (12), de las prácticas culturales (16) y aspectos fitopatológicos (7), etc. Sin embargo, la información existente en cuanto al almacenamiento de tubérculos-semillas es aún escasa $(2,8)$. Esto es particularmente cierto en cuanto a la modificación de las respuestas de los tubérculos almacenados en diferentes sistemas, ante el agregado de fertilizantes.

Si bien la influencia de los distintos sistemas de almacenamiento sobre la edad fisiológica y el rendimiento potencial de la "semilla" ha sido estudiada $(1,3)$, es muy limitado aquello que se conoce respecto a su interacción con la fertilización nitrogenada (5). 
Por otra parte, se sabe que el $\mathrm{N}$ ejerce un importante efecto en la regulación de la tuberización (11) y que los aumentos que se logran en el rendimiento por la aplicación de este nutriente, provienen de modificaciones del área foliar (10).

En la producción de tubérculo-semilla interesa que éstos sean plantados con una edad fisiológica adecuada; por diferentes causas esto no siempre se logra por lo que, es de gran interés conocer las posibilidades de modificar la respuesta de los tubérculos con distinta edad fisiológica. El objetivo de este trabajo ha sido evaluar los efectos de la aplicación del nitrógeno sobre la capacidad de cobertura foliar y el rendimiento.

\section{MATERIALES Y MÉTODOS}

El trabajo se llevó a cabo durante los años agrícolas 1985-86 y 198687, en el campo experimental de la Facultad de Agronomía de La Plata $\left(35^{\circ} \mathrm{S}\right)$, utilizando Spunta y Ballenera MAA, cultivares de características semitemprana y semitardía, respectivamente.

Los tubérculos se conservaron en cámaras refrigeradas a $4^{\circ} \mathrm{C}(\mathrm{F})$ y en luz natural difusa a temperatura ambiente (LND). El período de conservación fue de 100 días en 1985-86 y de 120 días en 1986-87. Previo a la plantación los tubérculos conservados en $\mathrm{F}$ se prebrotaron durante 30 días en LND. Luego del período de almacenamiento se evaluó la edad fisiológica de los tubérculos según la metodología propuesta por Caldiz et al. (2).

La plantación se realizó el 5/1 2/85 y el 10/1 2/86 con tubérculos enteros, en forma manual, en parcelas de 4 surcos y $4 \mathrm{~m}$ de largo a $0.70 \mathrm{~m}$ entre surcos y $0.30 \mathrm{~m}$ entre plantas. Todas las parcelas fueron fertilizadas con el equivalente a $200 \mathrm{~kg} / \mathrm{ha}$ de $\mathrm{P}_{2} \mathrm{O}_{5}$ y además con los siguientes niveles de N: No: testigo sin aplicación de nitrógeno; N,: 200 $\mathrm{kg} / \mathrm{ha}$ de nitrógeno en la plantación y $\mathrm{N}_{2}: 100 \mathrm{~kg} / \mathrm{ha}$ en plantación más $100 \mathrm{~kg} / \mathrm{ha}$ al inicio de la tuberización (45 días de la plantación). El suelo era un argiudol vértico.

El porcentaje de plantas emergidas se determinó a los 20 días de la plantación y durante el cultivo se evaluó la capacidad de cobertura foliar (CCF) según el CIP (4). A la cosecha, con base en 5 plantas por parcela se determinó el peso de tubérculos, considerando sólo todos aquéllos con un diámetro mayor a $3 \mathrm{~cm}$. La estimación de rendimiento por ha se realizó teniendo en cuenta el porcentaje de plantas emergidas. Los experimentos fueron conducidos en bloques al azar en factorial. Cada ensayo permitió combinar y analizar los dos sistemas de almacenamiento, los dos cultivares y las aplicaciones de $\mathrm{N}$ con tres 
repeticiones por tratamiento; las diferencias se establecieron por el test de Tukey (p: 0.05).

En la Figura 1 se presentan los datos climáticos durante los ensayos.

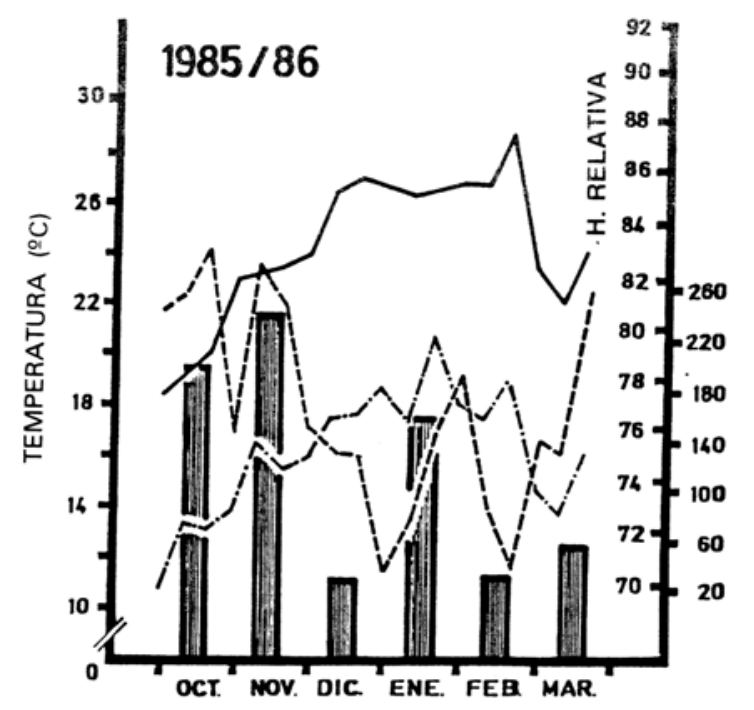

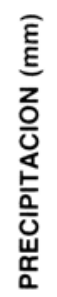

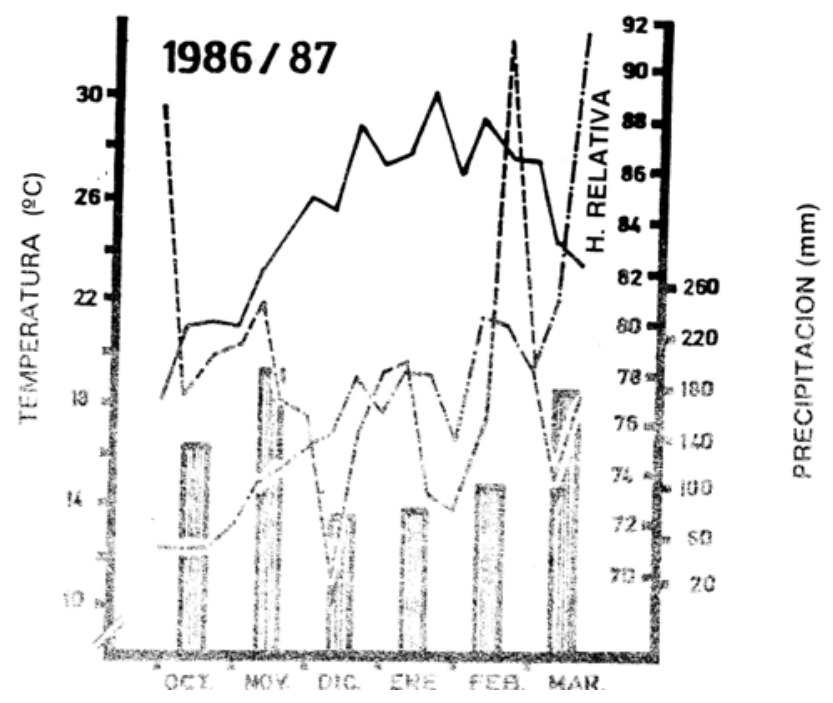

FIGURA 1. Datos climáticos durante los ensayos. ( IIII ) precipitaciones; ( $)$ temperatura media máxima y (-._._. - \} media mínima; $\left(-C_{-}\right)$ humedad relativa. 


\section{RESULTADOS Y DISCUSIÓN}

\section{Edad Fisiológica}

La conservación en LND determinó que los tubérculos alcanzaran la plantación con mayor edad fisiológica. Esto sucedió en los dos años para ambos cultivares (Tabla 1). Estos resultados confirman trabajos anteriores (1), donde la conservación en LND produjo tubérculos fisiológicamente más envejecidos que aquéllos conservados en $\mathrm{F}$. En 1986-87 existió una diferencia entre cultivares, debido a que Spunta es más sensible al envejecimiento fisiológico $(2,3)$.

TABLA 1. Efectos del sistema de almacenamiento. Frío (F) y Luz Natural Difusa (LND) sobre la edad fisiológica (en días).

\begin{tabular}{|c|c|c|c|c|}
\hline & \multirow{2}{*}{$\begin{array}{l}\text { 1985-86 } \\
\text { cv. Spunta }\end{array}$} & \multicolumn{3}{|c|}{ 1986-87 } \\
\hline & & cv. Spunta & & cv Ballenera \\
\hline \multicolumn{5}{|c|}{$\begin{array}{l}\text { Sistema de } \\
\text { Almacenamiento }\end{array}$} \\
\hline $\begin{array}{l}\text { Luz Natural } \\
\text { Difusa (LND) }\end{array}$ & $38 \quad b$ & $20 \quad b$ & * & $29 \mathrm{~b}$ \\
\hline Frío (F) & $46 \mathrm{a}$ & $27 \mathrm{a}$ & * & $35 \mathrm{a}$ \\
\hline
\end{tabular}

Dentro de una misma columna los valores seguidos por letras iguales no difieren entre sí. (*) Indica diferencia significativa entre cultivares (p:0.05).

\section{Emergencia}

En 1985-86 se registró una menor emergencia para ambos sistemas de conservación en relación con 1986-87. La falta de agua en el suelo en las etapas previas y durante la fase de preemergencia fue determinante de este comportamiento (Figura 1, Tabla 2). Las condiciones desfavorables determinaron que la emergencia de los tubérculos conservados en LND fuera un $25 \%$ mayor que los conservados en F. La presencia de un mayor número de raíces preformadas en los brotes de los tubérculos de LND y su mayor grado de envejecimiento, fueron los causantes de una mayor emergencia y establecimiento de los brotes. Es deseable una rápida emergencia y crecimiento del follaje, debido a que en la papa el tubérculo madre continúa siendo la fuente de asimilados, hasta que el área foliar alcanza los $300 \mathrm{~cm}^{2}$ (13). 
Por otra parte, dentro de cada sistema de almacenamiento el tratamiento sin adición de $\mathrm{N}$ (No) fue el que produjo los mayores porcentajes de emergencia. Probablemente, las condiciones de suelo seco en la plantación, más los altos niveles de $N_{1}(100 \mathrm{~kg} / \mathrm{ha}$ de $\mathrm{N})$ determinaron una sequía fisiológica, que influyó negativamente sobre el número de plantas emergidas. La reducción en el porcentaje de plantas emergidas fue mayor en F que en NLD, lo cual coincide con los resultados de trabajos anteriores.

TABLA 2. Efectos del sistema de almacenamiento (Frío y Luz Natural Difusa) y del momento de fertilización sobre la emergencia de plantas (en \%).

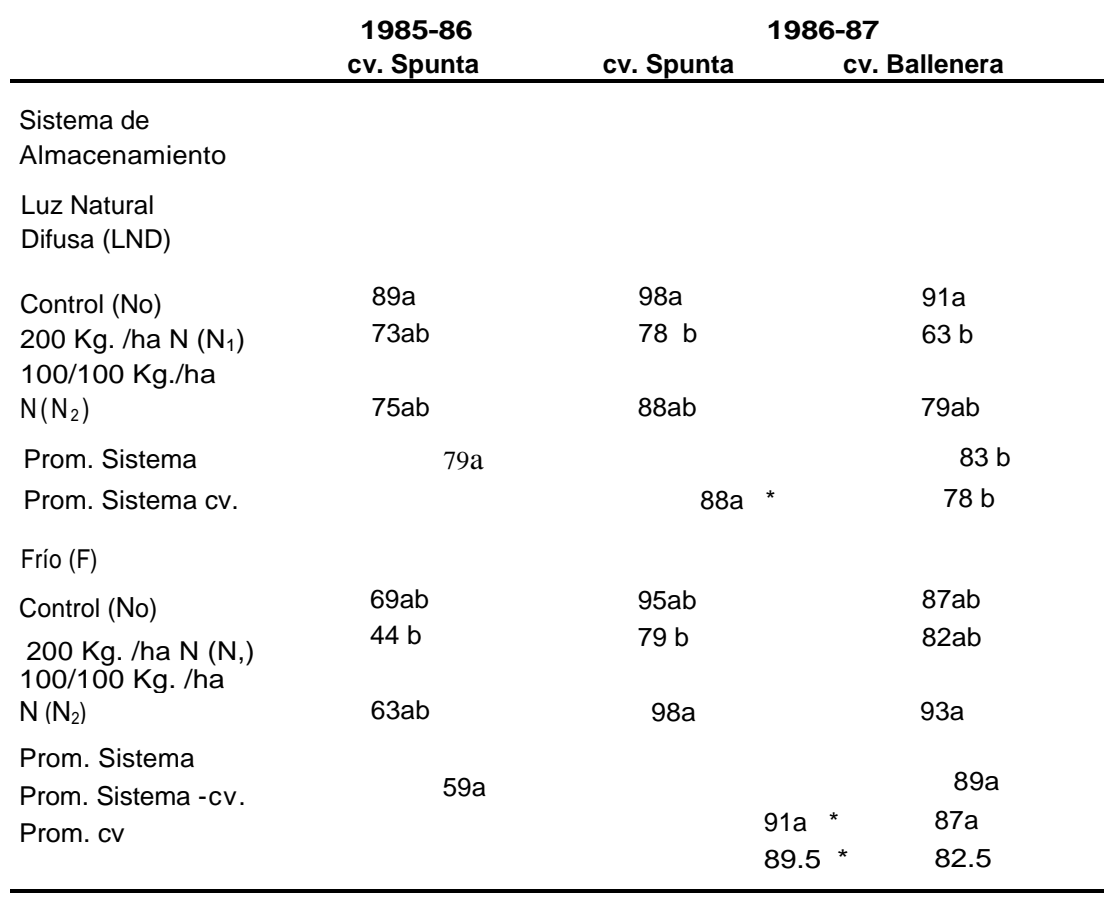

Dentro de una misma columna los valores seguidos por letras iguales no difieren entre si. $\left(^{*}\right)$ Indica diferencia significativa entre cultivares y sistemas - cultivares ( $p$ : 0.05).

En 1986-87 existieron diferencias significativas entre cultivares, observándose una mayor emergencia en Spunta; éste es un cultivar que normalmente registra una mayor capacidad de establecimiento de los brotes que ballenera MAA (14). en los tubérculos conservados en 
LND la emergencia fue menor que en F, y dentro de LND Spunta se comportó mejor que Ballenera MAA. En Spunta no existieron diferencias entre los sistemas de almacenamiento, pero sí en Ballenera MAA, donde se registró un incremento del $10 \%$ en la emergencia para los tubérculos conservados en $\mathrm{F}$. En el caso de Spunta, no hubo diferencias entre los sistemas, lo cual podría atribuirse a la mejor respuesta de este cultivar al sistema LND.

\section{Capacidad de Cobertura Foliar}

No hubo diferencias significativas entre cultivares ni tampoco entre sistemas al considerar la integración de los valores de CCF (Figura 2). De todos modos, existieron diferencias a lo largo del ciclo de cultivo a partir de la interacción entre los tratamientos con $\mathrm{N}$ y los sistemas de almacenamiento. En coincidencia con los resultados de Panelo et al. (14), el patrón de crecimiento de los cultivares fue diferente, tal como se deduce del comportamiento de los testigos donde Ballenera MAA mantuvo una mayor CCF que Spunta.

Spunta fue el cultivar que mejor respondió a la fertilización en los dos momentos de aplicación, particularmente en LND; en tanto que Ballenera MAA sólo respondió cuando el $\mathrm{N}$ se aplicó fraccionado (N2). Para este tratamiento hubo una mayor respuesta del material conservado en F, para Ballenera MAA y de LND para Spunta.

En Ballenera MAA el efecto del sistema de almacenamiento resultó menor que el de Spunta, dada su menor sensibilidad al envejecimiento (9). En este caso, los tubérculos conservados en LND mantuvieron su CCF en valores superiores a los de F, especialmente hacia el final del ciclo de cultivo.

La aplicación fraccionada de $\mathrm{N}\left(\mathrm{N}_{2}\right)$ produjo una mejor CCF en LND que en $\mathrm{F}$, probablemente por un mayor aprovechamiento del $\mathrm{N}$ al inicio del cultivo. En Ballenera, un cultivar de lento crecimiento (14), hubo un aumento en la CCF del testigo (No), en forma opuesta a Spunta, en la cual la CCF decayó sensiblemente durante todo el ciclo. Es probable que la diferencia en los patrones de crecimiento de estos cultivares estén ligadas a diferencias en la capacidad de absorción y aprovechamiento del $\mathrm{N}$, tal como lo sugieren Dwelle et al. (6). 


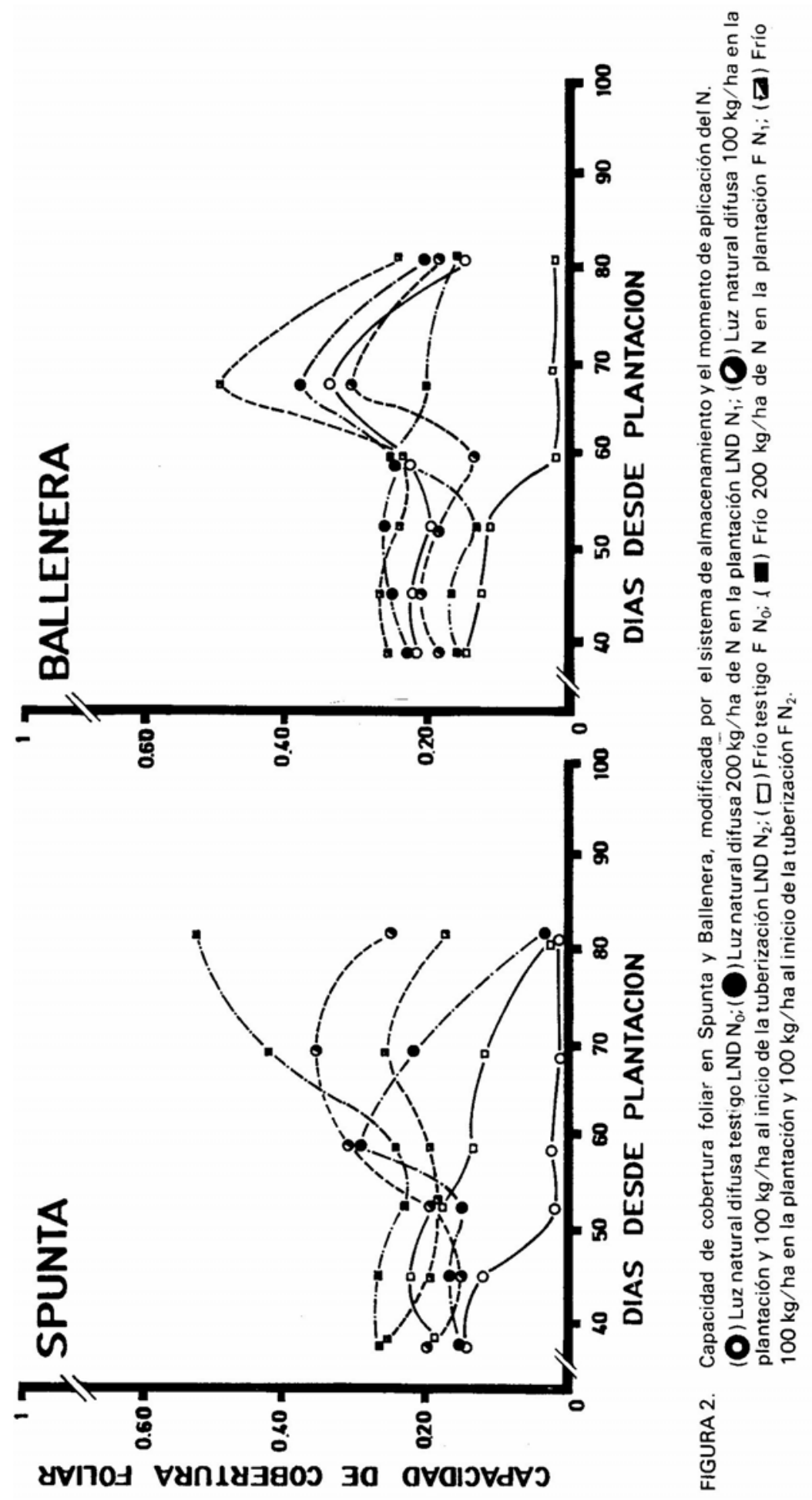


En líneas generales la CCF resultó menor en los tubérculos provenientes de LND, especialmente en Spunta, en coincidencia con de Vaz y Gunasena (5); estas diferencias en la CCF se reflejaron en Spunta, en el rendimiento de tubérculos.

\section{Rendimiento}

En 1985-86 no se registraron diferencias significativas en el rendimiento, aunque sí hubo una tendencia a incrementarlo en LND cuando se aplicó IM, (Tabla 3). En este año no existieron diferencias entre los sistemas de almacenamiento, pero en 1986-87 el sistema LND produjo menores rendimientos que F. Spunta produjo mayores rendimientos que Ballenera MAA, en coincidencia con los resultados de Panelo et al. (14), cuando los niveles de fertilidad fueron altos. En Spunta tanto en LND como en $\mathrm{F}$ la aplicación de $\mathrm{N}_{2}$ permitió lograr aumentos significativos en el rendimiento; pero en Ballenera MAA estas diferencias no existieron para LND, pero fueron significativas en $\mathrm{F}$, lográndose un incremento de rendimiento de $7 \mathrm{t} / \mathrm{ha}$.

TABLA 3. Efectos del sistema de almacenamiento (Frío y Luz Natural Difusa) y del momento de fertilización sobre el rendimiento de tubérculos (t/ha).

\begin{tabular}{|c|c|c|c|}
\hline & \multirow{2}{*}{$\begin{array}{l}\text { 1985-86 } \\
\text { cv. Spunta }\end{array}$} & \multicolumn{2}{|c|}{$1986-87$} \\
\hline & & cv. Spunta & cv. Ballenera \\
\hline \multicolumn{4}{|l|}{ Sistema de } \\
\hline \multicolumn{4}{|l|}{ Almacenamiento } \\
\hline \multicolumn{4}{|l|}{ Luz Natural } \\
\hline \multicolumn{4}{|l|}{ Difusa (LND) } \\
\hline Control (N0) & $11,38 a$ & $11,02 \mathrm{c}$ & $17,71 \mathrm{ab}$ \\
\hline $200 \mathrm{Kg}$. ha $N(N)$, & $14.33 a$ & $14,80 \mathrm{bc}$ & $16,39 a b$ \\
\hline \multicolumn{4}{|l|}{$100 / 100 \mathrm{Kg} . / \mathrm{ha}$} \\
\hline (N2) & $12.70 a$ & $21.88 \mathrm{~b}$ & $14,48 \mathrm{~b}$ \\
\hline Prom. Sistema & $12,80 a$ & & $16,04 \mathrm{~b}$ \\
\hline Prom. Sistema -cv. & & $15,90 b$ & $16,19 a$ \\
\hline \multicolumn{4}{|l|}{ Frío (F) } \\
\hline Control (No) & $12,94 a$ & $14.54 \mathrm{bc}$ & $12,55 b$ \\
\hline $200 \mathrm{Kg} . / \mathrm{ha} \mathrm{N}(\mathrm{N})$, & $13.51 \mathrm{a}$ & $16.37 \mathrm{bc}$ & $11,23 b$ \\
\hline \multicolumn{4}{|l|}{$100 / 100 \mathrm{Kg} . / \mathrm{ha}$} \\
\hline$\left(\mathrm{N}_{2}\right)$ & $13.89 a$ & $41,08 a$ & $19.37 a$ \\
\hline Prom. Sistema & $13.44 a$ & & $19,19 a$ \\
\hline Prom. Sistema - cv. & & $23,99 a$ & $14,38 a$ \\
\hline Prom. cv. & & 19,95 & 15,28 \\
\hline
\end{tabular}


Estas diferencias varietales son parte del conjunto de diferencias que definen a estos cultivares, e indudablemente también afectan la utilización del $\mathrm{N}$ de acuerdo con su edad fisiológica.

Considerando el efecto del sistema de almacenamiento, Spunta rindió en $\mathrm{F}$ un $60 \%$ más que Ballenera MAA. Esta diferencia radica especialmente, en la respuesta de Spunta a la aplicación fraccionada $\left(\mathrm{N}_{2}\right)$.

No existió en ninguno de los años y para ninguno de los cultivares, una correlación entre la edad fisiológica de los tubérculos y el rendimiento, tal como ha sido demostrado en otros trabajos $(1,15)$. Probablemente, la variabilidad de los resultados hayan influido sobre esta falta de correlación.

De los resultados obtenidos, se concluye que, la utilización de altas dosis de $\mathrm{N}$ en forma dividida, podría compensar en cuanto al rendimiento, el envejecimiento fisiológico en cultivares sensibles al mismo, como en Spunta. Estas modificaciones estarían dadas por aumentos en las CCF hacia el final del ciclo de cultivo.

Por otra parte, parece de interés continuar evaluando las distintas respuestas varietales en cuanto al sistema de almacenamiento y la fertilización, a fin de explicar más detalladamente los mecanismos de respuesta. Esto permitiría realizar un uso más eficiente de los insumos y lograr una mejor utilización de los cultivares, de acuerdo con su sensibilidad al envejecimiento fisiológico.

\section{Agradecimientos}

A los Ings. Agrs. Mabel Panelo (Chacra Experimental de Miramar) y V. Arrate (Asesores Agrarios, Balcarce) por la provisión de los tubérculos semillas.

Al personal de campo de la Facultad de Agronomía y a los Sres. H. Acciaresi, R. Bezus, J. J. Garat y S. D. Tenembaum por la colaboración brindada en la conducción de los ensayos.

\section{REFERENCIAS BIBLIOGRÁFICAS}

1. Caldiz, D. O. 1 989. Influence of origin and storage system on the physiological age, crop growth and tuber yield of seed potatoes. (En preparación).

2. Caldiz, D. O.; Claver. F. K., Escande, A. R. 1984. Effect of harvesting time and storage system on the quality of seed potato tubers (Solanum tuberosum L). Turrialba 34: 287-290. 
3. Caldiz, D. O.; Panelo, Mabel; Claver, F.K.; Montaldi E.R. 1985 The effects of two planting dates on the physiological age and yielding potential of seed potatoes grown in a warm temperature climate in Argentina. Potato Res. 28: 425-434.

4. Centro Internacional de la Papa. 1986. Método sencillo para interpretar el crecimiento y rendimiento del cultivo de papa. Circular del CIP 14: 7-9.

5. De Vaz, C. R.; Gunasena, H. M. P. 1976. Effect of physiological age of seed tubers, presprouting and time of nitrogen application on yield potato. Tropical Agricult. 132: 115-124.

6. Dwelle, R. B.; Kleinkopf, G. E.; Pavek, J. J.; Hurley, P. J. 1983. Breeding of potatoes for greater phosynthetic efficiency and greater nitrogen efficiency. Procc. Int. Cong. Research for the Potato in the Year 2000. International Potato Center, Lima, Perú. 76-77.

7. Escande, A. R.; Calderoni, A. V.; Melegari, A. L. 1984. La Papa. Diagnóstico y control de sus enfermedades. INTA, UNMDP, CIAM. Balcarce y Mar del Plata. $48 \mathrm{p}$.

8. Escande, A. R ; Melgari, A. L.; Caldiz, D. O.; Rodríguez, J. C.; Palladino, H.; Mirón, R. H. 1985. Influencia del sistema de almacenamiento sobre la capacidad simiente dé tubérculos de papa (Solanum tuberosum L. cvs. Spunta y Sierra Volcán). IDIA 437/440: 24-30.

9. Escande, A. R.; Caldiz, D. O.; Rodríguez, J. C. 1986. Influencia del sistema de almacenamiento y de la época de plantación sobre la productividad de tubérculos de papa (Solanum tuberosum L.) simiente. Turrialba 36: 237-244.

10. Harris, P. M. 1978. Mineral Nutrition. En: The Potato Crop. P. M. Harris, Ed. Chapman and Hall, London. 730 pp.

11. Krauss, A. 1985. Interaction of nitrogen nutrition, phytohormones and tuberization. En: Potato Physiology. P. H. Li, Ed. Acadernic Press Ind., Orlando. 209-231.

12. Mendiburu, A. O.; Lucarini, O. R. 1980. Manipulaciones genéticas para la producción y el aprovechamiento de la papa. Rev. Fac. Agron. Buenos Aires 1: 129-139.

13. Milthorpe, F. L.; Moorby, J. 1974. An introduction to Crop Physiology. Cambridge University Press. 202 p.p.

14. Panelo, Mabel; Caldiz. D. O.; Claver, F. K. 1982. La tuberización y el rendimiento de tres variedades de papa (Solanum tuberosum L.) cultivadas en Miramar, Argentina. Rev. Fac. Agron. La Plata 58: 99-120.

15. Panelo, Mabel; Caldiz, D. O. 1989. Influence of early haulm killing of seed crops on subsequent sprouting, physiological ageing and tuber yield. Potato Res. 32 (En prensa).

16. Rohatsch, P. 1983. Control de pulgones. 3ra. Jornada de Actualización Técnica: Papa. CIAM, INTA, Facultad de Ciencias Agrarias, Mar del Plata y Balcarce. 113-114. 